\title{
Helicopter Classification via Period Estimation and Time-Frequency Masks
}

\author{
Rui Zhang ${ }^{\# 1}$, Gang Li ${ }^{\# 2}$, Carmine Clemente ${ }^{* 3}$, Pramod K. Varshney ${ }^{\dagger 4}$

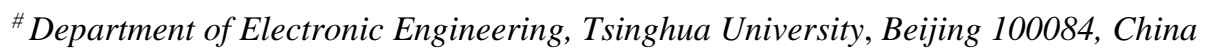 \\ ${ }^{1}$ z-r12@mails.tsinghua.edu.cn, ${ }^{2}$ gangli@mail.tsinghua.edu.cn \\ * University of Strathclyde, CeSIP, EEE, 204 George Street, G1 1XW, Glasgow, UK \\ 3 carmine.clementedstrath.ac.uk \\ ${ }^{\dagger}$ Department of Electrical Engineering and Computer Science, Syracuse University, Syracuse, NY 13244 USA \\ ${ }^{4}$ varshneydsyr.edu
}

\begin{abstract}
The rotation of blades of a helicopter induces a Doppler modulation around the main Doppler shift, which is commonly called the micro-Doppler signature and can be used for target classification. In this paper, an automatic helicopter classification method is proposed by estimating the period of the micro-Doppler signature and identifying the number of blades via time-frequency masks. The advantages of this method are threefold: (1) it determines the number of blades automatically; (2) it significantly reduces the computational burden compared to the classical model dictionary-based classification methods; $(3)$ it is robust with respect to the inclination of the helicopter. The effectiveness of the proposed approach is validated by using both synthetic and real data.
\end{abstract}

\section{INTRODUCTION}

In radar applications, mechanical vibration or rotation of a target or its parts introduces additional frequency modulation of the received signals. This phenomenon is referred to as the micro-Doppler effect [1]. Micro-Doppler parameters such as Doppler repetition period, Doppler amplitude, and initial phase directly indicate unique characteristics of the target and, therefore, can be exploited for civil and military purposes such as target classification and identification [2].

The radar return of a helicopter contains micro-Doppler components generated by its rotor blades, which provides important information to facilitate helicopter classification [3]-[6]. In [3], the $L / N$-quotient helicopter classification method is presented to classify helicopters, where $N$ denotes the number of blades and $L$ is the length of each blade. However, the $L / N$-quotient may be the same for two different helicopter models [3], and the value of $L / N$ is subject to the angle that the line of sight (LOS) forms with the plane on which the blades lie, which reduces the robustness of the $L / N$ quotient based method. A classification scheme based on time-frequency analysis is proposed in [4], where the microDoppler parameters are extracted in time-frequency domain. This method allows the identification of the parity of the number of blades, i.e., whether the number is even or odd, according to the time-frequency distribution of the flashes. But automatic classification is not considered in detail and some procedures still require manual intervention. In [5], helicopter classification using a high resolution continuous wave radar is addressed. It employs two matched masks in slow time-range domain to solve the classification problem where the two helicopter classes could be easily confused with each other. However, this method still requires estimation of the $L / N$-quotient, which is influenced by the inclination of the helicopter with respect to the LOS. In [6], the authors derive a maximum likelihood (ML) algorithm for estimating helicopter parameters, and several information theoretic criteria are considered for blade number selections.

Recently, considering that the micro-Doppler signatures of helicopter blades have sparse properties, a model-based classification algorithm was introduced in [7], where a sparse signal model for received helicopter echoes was designed and used in conjunction with a greedy sparse signal recovery algorithm to extract the micro-Doppler parameters. This method enables high classification accuracy without prior information about the inclination of the helicopter. However, it does not take the parity of the number of blades into consideration, which may cause ambiguity in classification processing. Moreover, this method employs a large dictionary of target models, thus its computational burden is rather heavy.

In this paper, we propose an automatic helicopter classification approach based on period estimation and timefrequency masks. Since the period is independent of the inclination of the helicopter, it is extracted as a feature for target classification. Then, inspired by the observation that the parity of $N$ can be identified in time domain and the value of $N$ can be determined in time-frequency domain, correlation coefficients and time-frequency tools are jointly used to determine the value of $N$. Finally, the target is classified based on the values of $N$ and $\omega$. The contributions of this paper are threefold: (1) compared to [3]-[5], [7], the proposed method determines the number of blades automatically; (2) compared to [6]-[7], the computational burden is significantly reduced; (3) compared to [3]-[5], our method is robust with respect to the inclination of the helicopter with respect to the LOS.

The remainder of the paper is organized as follows. In Section II, the background related to micro-Doppler signal model and time-frequency analysis is introduced. Then the proposed method is presented in detail in Section III. Experimental results on synthetic and real data are given in Section IV to validate the proposed approach. Concluding remarks are provided in Section V. 


\section{BACKGROUND}

\section{A. Signal Model}

Assuming that a helicopter has already been detected in a certain radar cell, and the micro-Doppler parameters $(N, \omega, L)$ represent the number, the rotational speed and the length, respectively, of the blades of the helicopter, the echoes from the blades can be expressed as [1]:

$$
\begin{aligned}
y\left(t_{m}\right)= & \sigma L \exp \left(-j \frac{4 \pi R_{0}}{\lambda}\right) . \\
& \sum_{n=0}^{N-1} \operatorname{sinc}\left[\Phi_{n}\left(t_{m}\right)\right] \exp \left[-j \Phi_{n}\left(t_{m}\right)\right], \\
& m=0,1, \ldots, M-1, \\
\Phi_{n}\left(t_{m}\right)= & \frac{2 \pi L}{\lambda} \cos \beta \cos \left(\omega t_{m}+\frac{2 \pi n}{N}+\theta\right),
\end{aligned}
$$

where $\lambda$ is the carrier wavelength of the radar system, $t_{m}$ denotes the sampling instant with sampling frequency $f_{s}, M$ is the length of the received signal, and $\operatorname{sinc}(\alpha)=\sin (\alpha) / \alpha . R_{0}$ is the range of the target and $\beta$ is the aviation angle, defined as the angle formed by the LOS and the plane on which the blades lie. In addition, $\sigma$ is the scattering coefficient of the blades, $\theta$ is a random phase which accounts for the initial position of the blades, and $\Phi_{n}\left(t_{m}\right)$ denotes the phase function of the echoes from the $n$-th blade.

The instantaneous frequency corresponding to the $n$-th blade can be directly obtained by taking the time derivative of $\Phi_{n}(t)$ :

$$
f_{m D, n}\left(t_{m}\right)=-\frac{\omega L}{\lambda} \cos \beta \sin \left(\omega t_{m}+\frac{2 \pi n}{N}+\theta\right),
$$

It is obvious from (3) that the maximum Doppler shift of the received signal is

$$
f_{m D, \max }=\frac{2 \omega L}{\lambda} \cos \beta
$$

To avoid frequency aliasing, the sampling frequency is set to $f_{s} \geq 2 f_{m D, \max }$. As depicted in (1) and (2), the received signal is periodic and the period is

$$
T=\frac{2 \pi}{N \omega}
$$

It can be seen from (4) and (5) that the $L / N$ quotient is related to $\beta$, thus the $L / N$ quotient-based classification scheme is not robust to the inclination of the helicopter. In contrast, it is obvious from (5) that $T$ is independent of $\beta$ and determined by $N$ and $\omega$. Based on this fact, $T$ is extracted as a target feature for classification in this paper.

\section{B. Time-frequency Analysis}

In order to represent the time-varying Doppler and magnitude behaviours of the received signals, the Short Time Fourier Transformation (STFT) of the received signal is employed in this paper [1]:

$$
\begin{gathered}
\operatorname{STFT}\left(t_{m}, f_{p}\right)=\sum_{\substack{k=0 \\
p=1}}^{M-1}\left(t_{k}\right) w\left(t_{k}-t_{m}\right) \exp \left(-j 2 \pi f_{p} t_{k}\right), \\
\text {. }
\end{gathered}
$$

where $w(t)$ is a window function, $P$ is the number of frequency bins. In comparison to the waveform and the spectrum, the time-frequency representation provides more details about the received signal. To show the superiority of time-frequency analysis, let us consider the following two target models: 1) $\mathbf{H}_{1}$, a two-bladed helicopter with parameters $\left.\left(N_{1}, \omega_{1}, L_{1}\right) ; 2\right)$ $\mathbf{H}_{2}$, a four-bladed helicopter with parameters $\left(N_{2}, \omega_{2}, L_{2}\right)=$ $\left(2 N_{1}, \omega_{1} / 2,2 L_{1}\right)$. According to (4) and (5), the received signals from $\mathbf{H}_{1}$ and $\mathbf{H}_{\mathbf{2}}$ have the same maximum Doppler shift $f_{m D \text {,max }}$ and period $T$. Therefore, the major signatures of $\mathbf{H}_{1}$ and $\mathbf{H}_{2}$ in time-domain and frequency-domain are highly similar to each other, and it is difficult to distinguish them by using waveform or spectrum analysis alone. However, since the received signals from $\mathbf{H}_{1}$ and $\mathbf{H}_{2}$ have different instantaneous frequencies, they are distinguishable in time-frequency domain.

\section{THE PROPOSED METHOD}

The proposed classification approach can be divided into four stages: A) synchronization; B) period estimation; C) blade number determination; and D) classification.

\section{A. Synchronization}

According to (1) and (2), the flashes of the received signal appear at those instants when one of the phase functions, i.e. $\Phi_{n}\left(t_{m}\right)$ equals $\pm \pi / 2$. In order to make the classification method independent of the initial phase of the blades, the instant $t=0$ is synchronized with the first flash of the received signal as described in [7].

\section{B. Period estimation}

In this paper, the value of $T$ is estimated by using the autocorrelation function of the $y\left(t_{m}\right)[1]$ :

$$
c\left(\tau_{k}\right)=\sum_{m=0}^{M-1-k} y\left(t_{m}\right) y^{*}\left(t_{m+k}\right), k=0,1, \ldots, M-1,
$$

where $\tau_{k}=k / f_{s}$, and $*$ denotes the conjugate operation. Because $y\left(t_{m}\right)$ is periodic with period $T$, the highest peak of $c(\tau)$ appears at $\tau=0$, and other peaks appear at $\tau=k T, k=1,2 \ldots$. Therefore, the period $T$ can be estimated by measuring the distance between the neighbouring peaks of the autocorrelation function.

\section{Blade number determination}

According to (5), after the period estimation, the value of $N \omega$ can be directly derived from $T$. Then the following two steps are carried out: first, the parity of $N$ is identified by using correlation coefficients in time-domain; second, the value of $N$ is determined by employing time-frequency masks.

\section{1) Identifying the parity of $N$}

We assume that the initial phase $\theta=\pi / 2$ and the period $T$ has been estimated, then the signal model in (1) can be rewritten as

$$
\begin{aligned}
y_{T, N, d}\left(t_{m}\right)=\text { const } & \sum_{n=0}^{N-1} \operatorname{sinc}\left\{-d \sin \left[\frac{2 \pi}{N}\left(\frac{t_{m}}{T}+n\right)\right]\right\} . \\
& \exp \left\{j d \sin \left[\frac{2 \pi}{N}\left(\frac{t_{m}}{T}+n\right)\right]\right\},
\end{aligned}
$$


where $d=2 \pi L \cos \beta / \lambda$, and the rotational speed $\omega$ is related to $N$ and $T$ according to (5). To measure the dependence between the received signal $y\left(t_{m}\right)$ and the signal model $y_{T, N, d}\left(t_{m}\right)$, the following correlation coefficients are calculated:

$$
c_{T, N}=\max \frac{\sum_{m=0}^{M-1} y_{T, N, d}\left(t_{m}\right) y^{*}\left(t_{m}\right)}{\sqrt{\sum_{m=0}^{M-1}\left|y_{T, N, d}\left(t_{m}\right)\right|^{2} \sum_{m=0}^{M-1}\left|y\left(t_{m}\right)\right|^{2}}}, d \in\left(0, \frac{N T f_{s}}{4}\right)
$$

where $d$ is set less than $N T f_{s} / 4$ so that $f_{m D \text {,max }}$ is less than $f_{s} / 2$ and frequency aliasing can be avoided. It is obvious that $c_{T, N}$ represents the maximum relevance between $y\left(t_{m}\right)$ and the signal model which has the same period $T$ as $y\left(t_{m}\right)$ and number of blades $N$.

TABLE I

SIMULATION PARAMETERS

\begin{tabular}{|l|l|}
\hline Parameter & Value \\
\hline$f_{s}$ & $8000 \mathrm{~Hz}$ \\
\hline$M$ & 2000 \\
\hline SNR & from $-10 \mathrm{~dB}$ to $10 \mathrm{~dB}$ with a step size of $2.5 \mathrm{~dB}$. \\
\hline$N$ & randomly selected from $\{2,4,6\}$ or $\{3,5,7\}$. \\
\hline$T$ & uniformly distributed in $[0.1,0.5] \cdot M / f_{s}$. \\
\hline$\omega$ & $2 \pi / N T$ \\
\hline$d$ & uniformly distributed in $[0.1,1] \cdot N T f_{s} / 4$. \\
\hline$\theta$ & $\pi / 2$ \\
\hline
\end{tabular}
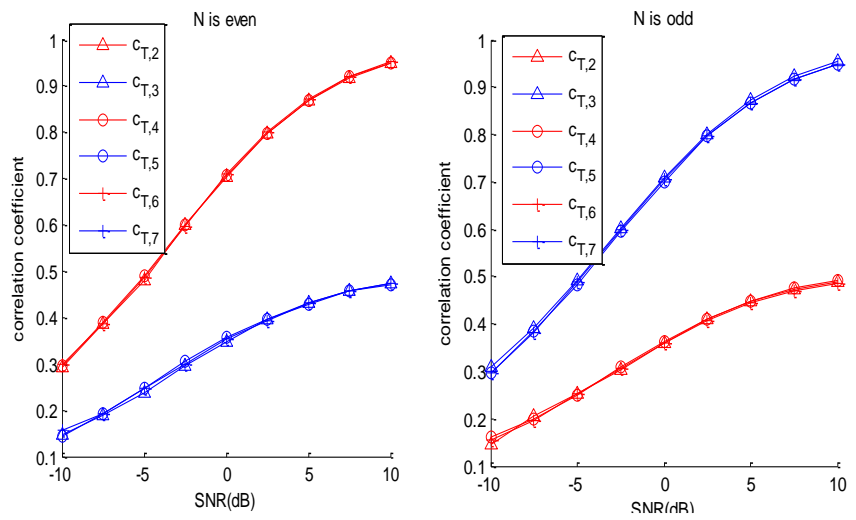

Fig. 1. Correlation coefficients between the received signal and the target models under different SNRs.

To demonstrate the properties of $c_{T, N}$, we perform a set of simulations. The received signal $y\left(t_{m}\right)$ is synthesized with parameters listed in TABLE I, and the correlation coefficients $\left\{c_{T, 2}, c_{T, 3}, \ldots, c_{T, 7}\right\}$ are calculated. The values of $\left\{c_{T, 2}, c_{T, 3}, \ldots\right.$, $\left.c_{T, 7}\right\}$ are averaged over 50 trials for each SNR, and the results are shown in Fig.1. Two conclusions can be obtained from Fig. 1: (1) $c_{T, 3} \approx c_{T, 5} \approx c_{T, 7}, c_{T, 2} \approx c_{T, 4} \approx c_{T, 6}$; (2) if $N$ is odd then $c_{T, 3}>c_{T, 2}$, else $c_{T, 3}<c_{T, 2}$.

Based on the above observations, we can identify the parity of $N$ by using the following algorithm:

Algorithm I: parity identification

input: synchronized signal $y\left(t_{m}\right)$ and its period $T$.

Step 1: calculate $c_{T, 2}$ and $c_{T, 3}$ according to (9).

Step 2: if $c_{T, 2}>c_{T, 3}$, then $N$ is even, otherwise $N$ is odd. output: parity of the number of blades.

\section{2) Determination of the value of $N$}

It is clear from Fig. 1 that the value of $N$ cannot be determined via correlation coefficients in time domain. Inspired by the fact that the instantaneous frequencies of different targets are distinguishable, time-frequency masks are employed. We assume that $\theta=\pi / 2, T$ has been estimated and $d$ has been calculated according to (9), then the timefrequency masks are configured as the STFT of $y_{T, N, d}$, denoted as $\operatorname{STFT}_{T, N, d}\left(t_{m}, f_{p}\right)$. Denoting the STFT of the received signal $y\left(t_{m}\right)$ as $\operatorname{STFT}\left(t_{m}, f_{p}\right)$, the correlation coefficients are calculated in the time-frequency domain:

$$
b_{T, N, d}=\frac{\sum_{p=0}^{P-1} \sum_{m=0}^{M-1} \operatorname{STFT}_{T, N, d}\left(t_{m}, f_{p}\right) \operatorname{STFT}^{*}\left(t_{m}, f_{p}\right)}{\sqrt{\sum_{p=0}^{P-1} \sum_{m=0}^{M-1}\left|S T F T_{T, N, d}\left(t_{m}, f_{p}\right)\right|^{2} \sum_{p=0}^{P-1} \sum_{m=0}^{M-1}\left|\operatorname{STFT}\left(t_{m}, f_{p}\right)\right|^{2}}},
$$

Then the value of $N$ can be determined as follows:

$$
N=\underset{N}{\arg \max }\left|b_{T, N, d}\right|
$$

It is worth emphasizing that: 1) since the parity of $N$ has been identified, only those numbers with the same parity as $N$ need to be considered in $(11) ; 2$ ) when calculating the values of STFT, the flashes of $y_{T, N, d}\left(t_{m}\right)$ and $y\left(t_{m}\right)$ should be cut off to avoid interference.

\section{Classification}

At the end of the first three steps, the period $T$ and the number of blades $N$ have been estimated. Let $\left(N_{v}, \omega_{v}\right)$ $(\nu=1,2, \ldots, V)$ be the parameters corresponding to $V$ known helicopters, the classification is obtained based on $T$ and $N$ :

$$
\hat{v}=\underset{v}{\arg \min }\left|T-\frac{2 \pi}{N_{v} \omega_{v}}\right|, \text { s.t. } N_{v}=N .
$$

The parameter $L$ and $\beta$ are not used in (12), but they have to be kept in the model in order to evaluate both $N$ and $\omega$.

\section{E. Remarks}

It can be seen from (10) that the time-frequency mask algorithm proposed in this paper belongs to matched filtering methods, which is optimum processing for a maximum output signal-to-noise ratio criterion as presented in [8]. In addition, several time-frequency tools besides STFT, such as WignerVille distributions (WVD), can also be used in (10) as discussed in [9].

\section{RESULTS}

\section{A. The Results with Simulated Data}

The algorithm is tested by simulated data. For different values of the SNR, 90 trial radar signals with a carrier frequency of $5 \mathrm{GHz}$ are generated, 10 for each target listed in TABLE II. The values of $\theta$ and $\beta$ are considered to be unknowns and are chosen randomly, in particular the latter ranges in $\left[0^{\circ}, 70^{\circ}\right]$. The duration and the sampling frequency are chosen equal to $0.25 \mathrm{~s}$ and $8 \mathrm{kHz}$, respectively. When calculating the STFT, a 32-points Hanning window is used and the number of frequency bins is set to be 64 . 
The performance in terms of classification accuracy is shown in Fig. 2. Even at $\mathrm{SNR}=-10 \mathrm{~dB}$, the helicopters are correctly classified with an accuracy of $82.2 \%$ It is clear that the classification accuracy of the proposed method is better than that of the method proposed in [7].

TABLE II

TARGET PARAMETERS

\begin{tabular}{|l|l|l|l|}
\hline Name & $\boldsymbol{N}$ & $\boldsymbol{\omega}$ & $\boldsymbol{L}$ \\
\hline AH-1 Cobra & 2 & 7.32 & 4.9 \\
\hline Mil MI-2 Hoplite & 3 & 3.30 & 4.1 \\
\hline AH-64 Apache & 4 & 7.32 & 4.8 \\
\hline UH-60 Black Hawk & 4 & 8.18 & 4.3 \\
\hline SA365 Dauphin & 4 & 5.97 & 5.8 \\
\hline A109 Agusta & 4 & 5.50 & 6.4 \\
\hline AS332 Super Puma & 4 & 7.80 & 4.4 \\
\hline MD 500E Defender & 5 & 4.03 & 8.2 \\
\hline CH-53 Stallion & 7 & 12.04 & 2.9 \\
\hline
\end{tabular}

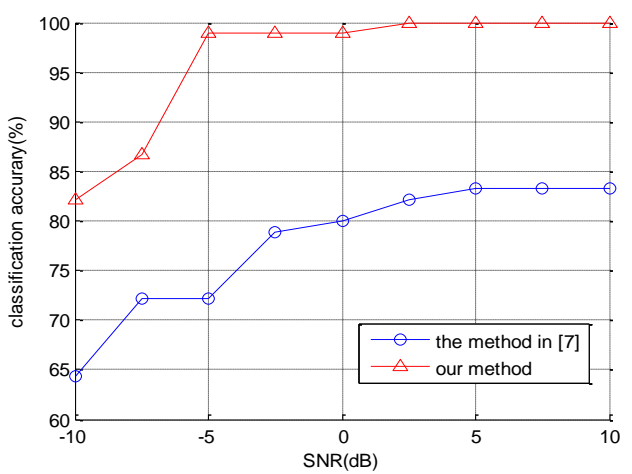

Fig. 2. Classification accuracies of our method and the method proposed in [7] versus SNR.

TABLE III

TARGET PARAMETERS

\begin{tabular}{|l|l|l|}
\hline Target & $\boldsymbol{N}$ & $\boldsymbol{\omega}$ \\
\hline A & 2 & 9.12 \\
\hline B & 2 & 12.42 \\
\hline C & 3 & 9.12 \\
\hline D & 3 & 12.42 \\
\hline E & 3 & 9.12 \\
\hline F & 3 & 12.42 \\
\hline G & 2 & 7.92 \\
\hline H & 2 & 10.77 \\
\hline
\end{tabular}

\section{B. The Results with Real Data}

The validity of the approach is also shown with real data. Signals from the two-bladed helicopter scale model GAUI X3 are acquired with a $24 \mathrm{GHz}$ radar. The database which contains the parameters of the helicopters to classify is shown in TABLE III: targets A and B correspond to the observed models; targets from $\mathrm{C}$ to $\mathrm{H}$ present either equal rotation speeds but different number of blades or the same number of blades with similar rotation speeds of the true targets A and B, in order to test the reliability of the algorithm. The signal length and the sampling frequency are chosen equal to $0.4 \mathrm{~s}$ and $5.5 \mathrm{kHz}$, respectively. Three data acquisitions are made for each speed, at three different aviation angles $\beta$. From each acquisition, whose total length is 20 seconds, 50 segments of
0.4 seconds are extracted as the inputs of the classification algorithm. The parameters used in the STFT are the same as that in Section IV Part A.

TABLE IV

CLASSIFICATION ACCURACY

\begin{tabular}{|l|l|l|l|}
\hline Target & $\boldsymbol{\beta}=\mathbf{0}^{\circ}$ & $\boldsymbol{\beta}=\mathbf{3 0}^{\circ}$ & $\boldsymbol{\beta}=\mathbf{6 0}^{\circ}$ \\
\hline A & $98 \%$ & $100 \%$ & $100 \%$ \\
\hline B & $100 \%$ & $100 \%$ & $100 \%$ \\
\hline
\end{tabular}

The rates of successful classification are shown in TABLE IV, which clearly demonstrate the effectiveness of out method.

\section{CONCLUSION}

In this paper, a novel method for helicopter classification was presented based on period estimation and micro-Doppler masks. The period of the received signal was estimated via the autocorrelation function and used to derive the product of the rotational speed and number of blades. Then the parity and the value of the number of blades were identified by using correlation coefficients and time-frequency masks, respectively. Finally, classification was accomplished based on the rotational speed and number of blades. This method determines the number of blades automatically and is independent of the inclination of the helicopter. Experiments with both synthetic and real data confirm the validity of the proposed method.

\section{ACKNOWLEDGMENT}

This work was supported in part by the National Natural Science Foundation of China under Grants 61422110 and 41271011, and in part by the Engineering and Physical Sciences Research Council (EPSRC) under Grant number EP/K014307/1 and the MOD University Defence Research Collaboration in Signal Processing.

\section{REFERENCES}

[1] V. C. Chen, The micro-Doppler effect in radar, Norwood, MA, USA: Artech House, 2011.

[2] C. Clemente, A. Balleri, K. Woodbridge, and J. Soraghan, "Developments in target micro-Doppler signatures analysis: radar imaging, ultrasound and through-the-wall radar," EURASIP Journal on Advances in Signal Processing, vol. 2013, no. 1, 2013.

[3] C. E. Rotander, H. V. Sydow, "Classification of helicopters by the L/N-quoutient," in Proceedings of the Radar 97, 1997, pp. 629-633.

[4] S. H. Yoon, B. Kim, and Y. S. Kim, "Helicopter classification using time-frequency analysis," IET Electronics Letters, vol. 36, no. 22, pp. 1871-1872, 2000.

[5] J. Muoz Ferraras, F. Perez Martinez, M. Burgos Garcia, "Helicopter classification with a high resolution LFMCW radar," IEEE Transactions on Aerospace and Electronic Systems, vol. 45, no. 4, pp. 1373-1384, 2009.

[6] P. Setlur, F. Ahmad, M. Amin, "Helicopter radar return analysis: Estimation and blade number selection," Signal Processing, vol. 91, no. 6, pp. 1409-1424, 2011.

[7] D. Gaglione, C. Clemente, F. Coutts, G. Li, and J. Soraghan, "ModelBased Sparse Recovery Method for Automatic Classification of Helicopters," in IEEE International Radar Conference, 2015.

[8] M. D. Flaska, "Cross correlation of short-time spectral histories," The Journal of the Acoustical Society of America, vol. 59, no. 2, pp. 381388, 1976.

[9] P. Flandrin, "On detection-estimation procedures in the time-frequency plane," in IEEE International Conference on Acoustics, Speech, and Signal Processing, 1986. 